\title{
Abnormal expression of multiple proteins predicts cancer-specific mortality in patients with high-grade non-muscle-invasive bladder cancer treated with transurethral resection
}

\author{
HIDEYASU TSUMURA ${ }^{1}$, KAZUMASA MATSUMOTO ${ }^{1}$, YUICHI SATO ${ }^{2}$, MASAOMI IKEDA ${ }^{1}$, \\ TETSUO FUJITA $^{1}$, TAKEFUMI SATOH ${ }^{1}$ and MASATSUGU IWAMURA ${ }^{1}$ \\ ${ }^{1}$ Department of Urology, Kitasato University School of Medicine; ${ }^{2}$ Department of Molecular Diagnostics, \\ Kitasato University School of Allied Health Sciences, Sagamihara, Kanagawa 252-0374, Japan
}

Received June 28, 2012; Accepted February 26, 2013

DOI: $10.3892 / \mathrm{mco} .2013 .92$

\begin{abstract}
High-grade non-muscle-invasive bladder urothelial carcinoma leads to various outcomes. It can cause death even after radical cystectomy and is treated only by transurethral resection (TUR). In the present study, we aimed to determine whether the molecular markers E-cadherin, coxsackie adenovirus receptor (CAR), S100A4 and uroplakin III are associated with clinicopathological outcomes in patients with high-grade non-muscle invasive bladder cancer (NMIBC) treated with TUR. Immunohistochemical staining was performed on serial sections from specimens obtained from 77 patients. Expression patterns were stratified according to the number of abnormally expressed markers: $0-1$ or $\geq 2$. The median follow-up time was 56 months (range, 3-287). The results from the present study indicated that expression of E-cadherin, CAR, S100A4 and uroplakin III was abnormal in 16, 17, 27 and $61 \%$ of tumors, respectively. Results of the log-rank test revealed that patients with abnormal expression of multiple molecular markers had a significantly increased risk of bladder cancer-specific mortality $(\mathrm{P}=0.016)$. The 5-year cancer-specific survival rates were 91 and $66 \%$ for patients with $0-1$ and $\geq 2$ molecular markers, respectively. No individual marker was associated with disease prognosis. Multivariate models that included clinicopathological outcomes and classified molecular markers indicated that abnormal expression of multiple molecular markers and lack of bacillus Calmette-Guérin (BCG) instillation are predictors of cancer-specific death ( $\mathrm{P}=0.046$ and 0.029 , respectively). Abnormal expression of multiple molecular markers is a strong predictor of mortality in bladder cancer patients undergoing TUR, suggesting that high-grade non-muscle-invasive cancer
\end{abstract}

Correspondence to: Dr Hideyasu Tsumura, Department of Urology, Kitasato University School of Medicine, 1-15-1 Kitasato Minami-ku, Sagamihara, Kanagawa 252-0374, Japan

E-mail: sugan@pd5.so-net.ne.jp

Key words: bladder cancer, high-grade, non-muscle-invasive bladder cancer, survival, immunohistochemistry is characterized by a variety of pathophysiological pathways. A combination of molecular markers may be useful in a minimally invasive modality for determining prognosis.

\section{Introduction}

High-grade non-muscle invasive bladder cancer (NMIBC) leads to various outcomes. It can cause death even after radical cystectomy and is treated only by transurethral resection (TUR) with or without intravesical therapy $(1,2)$. The ability to predict metastasis or disease progression is essential for selection of the optimal treatment for patients who have high-grade NMIBC. The prognostic value of traditional clinical and histopathological features such as tumor size and number as well as carcinoma in situ is well-documented (2-4). However, for patients with a primary diagnosis of high-grade NMIBC selection between conservative management and early cystectomy following diagnostic TUR remains a challenge.

A variety of molecular markers for progressive bladder cancer have been investigated (5-7). However, molecular markers that predict the progression of NMIBC are currently not well-documented and none is routinely used in clinical practice. The loss of E-cadherin expression has been associated with progression of malignant neoplasms through loss of cell-cell contact and is assumed to be crucial in the progression from a tumor in situ to an invasive and metastasizing lesion in various types of cancer $(8,9)$. The expression of coxsackie adenovirus receptor (CAR) has been associated with cell-cycle regulation and may be associated with effector proteins to elicit the growth inhibitory signal cascade (10). S100A4 is secreted from tumor cells and has a significant stimulatory effect on angiogenesis (11). Uroplakin III plays a key role in urothelial functions, including participation in the permeability barrier, adjustment of urothelial surface area, stabilization of the urothelial surface and development of the urinary tract (12). Previous studies conducted by our group showed that the expression of E-cadherin, CAR, S100A4 and uroplakin III is associated with disease progression and cancer-specific death in patients undergoing radical cystectomy with muscle-invasive bladder cancer or who were resistant to conservative treatment $(6,7,13)$. However, whether these markers are associated with 
disease progression in cases of high-grade NMIBC remains to be fully elucidated.

The aim of the present study was to determine the association of abnormal expression of E-cadherin, CAR, S100A4 and uroplakin III with pathologic characteristics and clinical outcome of high-grade NMIBC in a well-defined set of patients with long-term follow-up who are at risk for bladder cancer-specific death. The availability of the expression status of the four markers in the same set of patients provided a unique opportunity to determine whether abnormal expression of E-cadherin, CAR, S100A4 and uroplakin III exerts a synergistic effect on bladder cancer survival.

\section{Materials and methods}

Patients. Seventy-seven patients diagnosed with high-grade NMIBC at initial TUR conducted in our institution between May, 1985 and December, 2006 were included in this retrospective study. The muscle layer of all the included patients was histologically confirmed in the initial TUR specimens and the tumors did not invade into the muscle layer. The median follow-up time from initial TUR was 56 months (range, 3-287). According to the WHO classification (1973) and the tumor-node-metastasis (TNM) system (2002), a grade 3 Ta-T1 tumor was evaluated as high-grade NMIBC. Routine laboratory investigations, chest X-ray and pelvic computed tomography or magnetic resonance imaging were performed and all the patients were found to have clinically localized NMIBC. The median patient age was 67 years (mean, 66.7; range, 36-91). Ten patients $(13 \%)$ underwent immediate cystectomy as their primary form of treatment within 3 months of the diagnostic TUR. Sixty-seven $(87 \%)$ patients initially retained their bladder, from which 44 were administered a 6 -week course of intravesical bacillus Calmette-Guérin (BCG) without maintenance therapy. Of the 67 patients, 7 underwent late cystectomy for persistent disease $(n=2)$, cancer progression $(n=2)$ or $B C G$ intolerance $(n=3)$.

A postoperative follow-up examination was scheduled every 3 and 4 months following TUR and cystectomy, respectively, for the first year, semiannually in the second year and annually thereafter, with more frequent examinations being scheduled when clinically indicated. No patient underwent prior radiation or systemic chemotherapy prior to cystectomy or TUR. Approval was granted by the Ethics Committee of Kitasato University Hospital (Sagamihara, Japan).

Immunohistochemistry. Immunohistochemical staining for E-cadherin, CAR, S100A4 and uroplakin III using serial sections from the same paraffin-embedded blocks was performed. Staining and scoring protocols for these proteins have been described previously $(6,13,14)$. Briefly, E-cadherin expression was evaluated as normal (90-100\% positive cells with preserved cell border staining) or abnormal, which included negative (0-10\% positive cells) and various degrees of heterogeneous expression (11-89\% positive cells). Regarding CAR, S100A4 and uroplakin III, each image was interpreted for immunoreactivity by using a $0-3+$ semiquantitation system for intensity of staining and the percentage of positive cells (labeling frequency percentage). The intensity grading scale ranged from no detectable signal, i.e., 0, to a strong signal observed at low-power magnification, i.e., 3. Labeling frequency was scored as $0(0 \%), 1(1-33 \%), 2(34-66 \%)$ or $3(67-100 \%)$. The sum index was obtained by totaling the scores of intensity and percentage. Expression scores of CAR and uroplakin III were stratified further into normal $(\geq 1)$ and abnormal (0). Expression scores of S100A4 were stratified further into normal (0-1) and abnormal (2s) for the purpose of presentation. Slides were reviewed by a single pathologist (Y.S.) who was blinded to the clinical and pathologic data. Normal urothelium was obtained as a control from a patient with no history of bladder tumor who underwent autopsy.

Statistical analysis. For the purpose of analysis, gender, tumor pathologic stage (pTa vs. pT1), tumor size ( $\leq 1 \mathrm{vs} .>1 \mathrm{~cm}$ ), number of tumors (single vs. multiple) and BCG instillation status (instillation vs. no instillation) were evaluated as dichotomous variables. Fisher's exact and the Chi-square tests were used to evaluate the association of the expression of the four molecular markers and clinicopathological characteristics, including gender, pathologic stage, tumor size, number of tumors and BCG instillation status. The Kaplan-Meier method was used to calculate survival functions and differences were assessed with the log-rank statistic. Univariate and multivariable survival analyses were performed with the Cox proportional hazards regression model, controlling for gender, pathologic stage, tumor size, number of tumors, the status of BCG instillation and protein expression ( $0-1$ vs. $\geq 2$ markers) as dichotomous variables. $\mathrm{P}<0.05$ was considered to indicate a statistically significant difference. All the reported P-values were two-sided. Analyses were performed using SPSS, version 11.0 for Windows (SPSS, Inc., Chicago, IL, USA) and Microsoft Excel (Microsoft, Redmond, WA, USA).

\section{Results}

Association of molecular markers with clinicopathological characteristics. Representative results of the immunohistochemistry for four molecular markers in cancerous bladder tissues are shown in Fig. 1. Normal urothelium was used for representative normal staining of each molecular marker as a control. Abnormal expression of E-cadherin, CAR, S100A4 and uroplakin III was noted in 12 (16\%), 13 (17\%), 21 (27\%) and $47(61 \%)$ patients, respectively. Of the 77 patients, $17(22 \%)$ had tumors with normal expression of all the molecular markers and one $(1.3 \%)$ had abnormal expression of all the molecular markers. The clinicopathological characteristics of the 77 patients and the association with the four molecular markers are provided in Table I. Abnormal expression of CAR was associated with the number of tumors $(\mathrm{P}=0.029)$.

Association of molecular markers with clinical outcomes. Twenty-four patients (31\%) succumbed to the disease at the time of the analysis (median time to death, 25 months; range, 5-142). The cause of death in 15 (19\%) patients was defined as advanced metastatic bladder cancer (median time to death, 21 months; range, 5-142), while 9 (12\%) patients died of intercurrent causes without evidence of disease progression. The cause-specific survival (CSS) rate in 77 patients after a follow-up of 5 years was $82 \%$. Thirty-five $(45 \%)$ patients who were treated with initial TUR with or without intravesical 


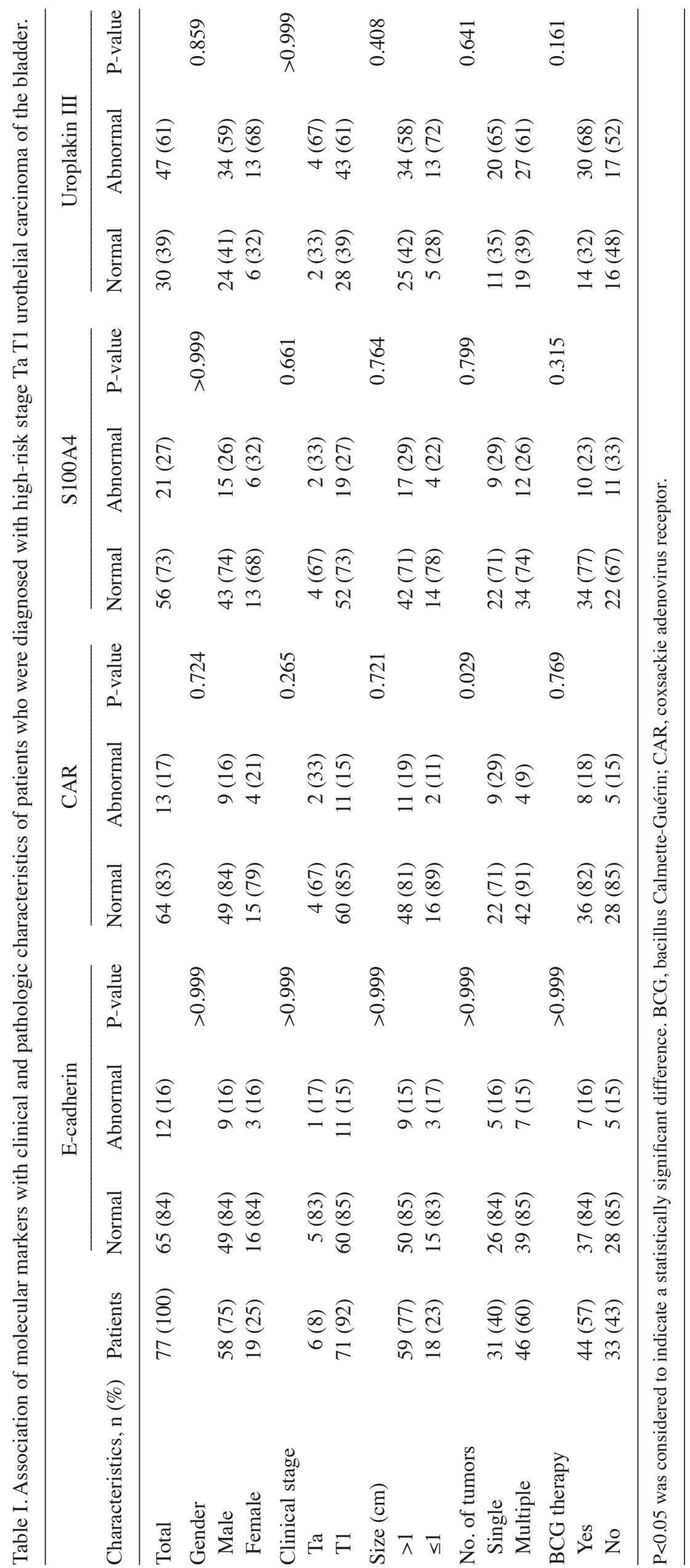



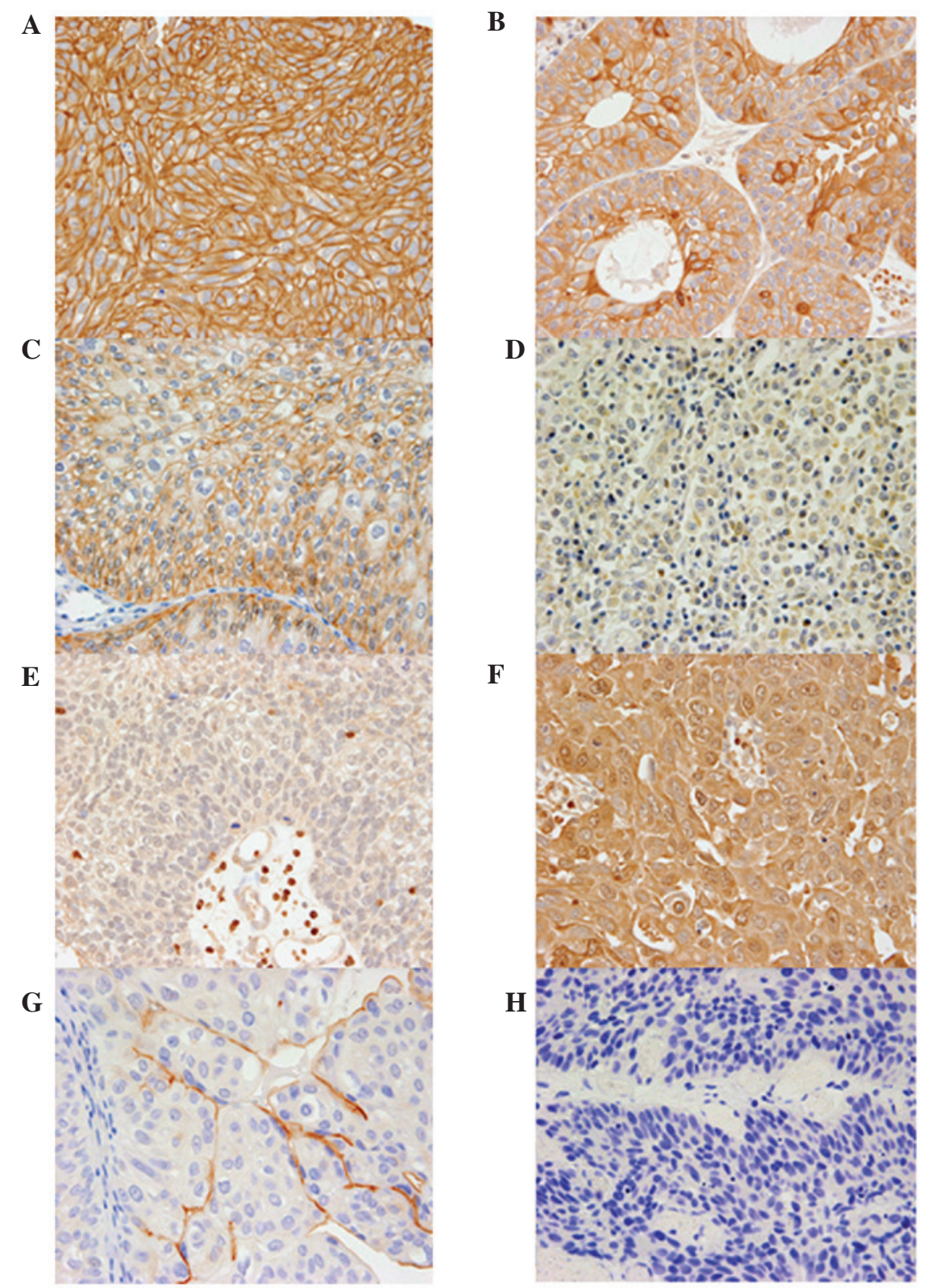

Figure 1. Representative E-cadherin, coxsackie adenovirus receptor (CAR), S100A4 and uroplakin III immunohistochemical staining in grade 3 non-muscle-invasive bladder cancer specimens (magnification, $\mathrm{x} 400$ ). Normal urothelium was used for representative staining of each molecular marker as a control. (A) Positive (normal) staining of E-cadherin in normal urothelium. (B) Negative (abnormal) staining of E-cadherin in tumor. (C) Positive (normal) staining of CAR in normal urothelium. (D) Negative (abnormal) staining of CAR in tumor. (E) Negative (normal) staining of S100A4 in normal urothelium. (F) Positive (abnormal) staining of S100A4 in tumor. (G) Positive (normal) staining of uroplakin III in normal urothelium. (H) Negative (abnormal) staining of uroplakin III in tumor.

A

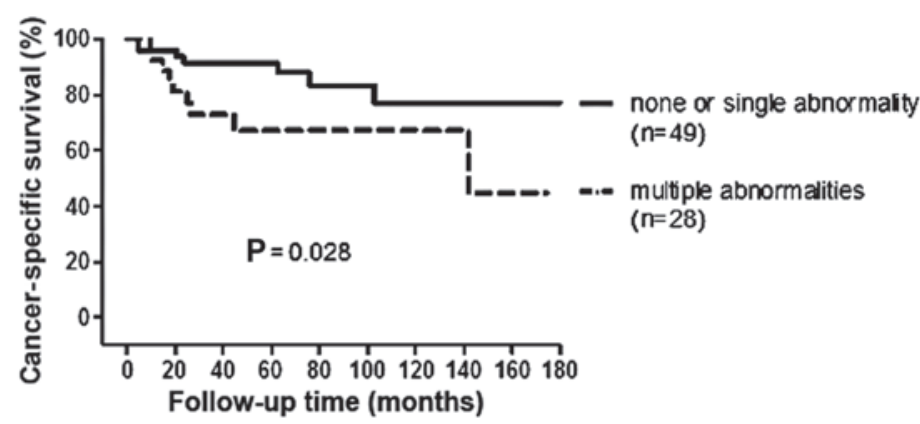

B

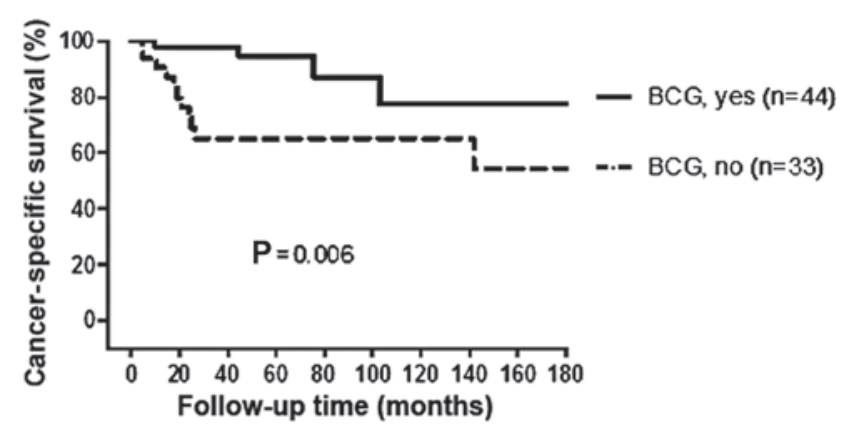

Figure 2. Probability of cancer-specific survival following initial transurethral resection according to the number of (A) molecular markers abnormally expressed and (B) bacillus Calmette-Guérin (BCG) intravesical treatment. 
Table II. Results of the univariate (UVA) and multivariate (MVA) Cox regression hazard model for cancer-specific survival in high-grade non-muscle-invasive bladder cancer.

\begin{tabular}{|c|c|c|c|c|c|c|}
\hline \multirow[b]{2}{*}{ Characteristics } & \multicolumn{3}{|c|}{ UVA } & \multicolumn{3}{|c|}{ MVA } \\
\hline & HR & $95 \%$ CI & P-value & HR & $95 \%$ CI & P-value \\
\hline \multicolumn{7}{|l|}{ Gender } \\
\hline Male & 1.000 & (Reference) & & 1.000 & (Reference) & \\
\hline Female & 0.433 & $0.097-1.922$ & 0.270 & 0.509 & 0.110 to 2.350 & 0.387 \\
\hline \multicolumn{7}{|l|}{ Stage } \\
\hline $\mathrm{Ta}$ & 1.000 & (Reference) & & 1.000 & (Reference) & \\
\hline $\mathrm{T} 1$ & 1.274 & $0.167-9.699$ & 0.814 & 2.120 & $0.211-21.28$ & 0.523 \\
\hline \multicolumn{7}{|l|}{ Tumor size $(\mathrm{cm})$} \\
\hline$\leq 1$ & 1.000 & (Reference) & & 1.000 & (Reference) & \\
\hline$>1$ & 0.786 & $0.250-2.475$ & 0.682 & 0.589 & $0.151-2.288$ & 0.445 \\
\hline \multicolumn{7}{|l|}{ No. of tumors } \\
\hline Single & 1.000 & (Reference) & & 1.000 & (Reference) & \\
\hline Multiple & 1.318 & $0.449-3.866$ & 0.615 & 1.317 & $0.418-4.145$ & 0.637 \\
\hline \multicolumn{7}{|l|}{ BCG therapy } \\
\hline Yes & 1.000 & (Reference) & & 1.000 & (Reference) & \\
\hline No & 4.291 & $1.361-13.53$ & 0.012 & 3.665 & $1.140-11.78$ & 0.029 \\
\hline \multicolumn{7}{|c|}{ No. of abnormal markers } \\
\hline 0 or 1 & 1.000 & (Reference) & & 1.000 & (Reference) & \\
\hline 2,3 or 4 & 3.344 & $1.176-9.523$ & 0.023 & 2.915 & $1.017-8.333$ & 0.046 \\
\hline
\end{tabular}

$\mathrm{P}<0.05$ was considered to indicate a statistically significant difference. HR, hazard ratio; $\mathrm{CI}$, confidence interval; BCG, bacillus Calmette-Guérin.

therapy did not have any evidence of tumor in a retained bladder at the time of analysis (median follow-up time, 59 months; range, 8-165).

The log-rank test results indicated that, compared with the abnormal expression of 0 or 1 molecular marker, the abnormal expression of multiple $(\geq 2)$ molecular markers was associated with an increased risk of bladder cancer-specific mortality ( $\mathrm{P}=0.028$, Fig. 2A). The 5-year CSS rate was 91 and $66 \%$ for $0-1$ and $\geq 2$ abnormal markers, respectively. BCG instillation was associated with a decreased risk for bladder cancer-specific mortality ( $\mathrm{P}=0.006$, Fig $2 \mathrm{~B})$. The 5-year CSS rate was 94 and $65 \%$ for patients with and without BCG instillation, respectively. Abnormal expression of any single molecular marker was not associated with increased risk of bladder cancer-specific mortality when the log-rank test was performed (data not shown).

Univariate and multivariate analyses by Cox regression hazard model were performed for CSS (Table II). The absence of BCG instillation and abnormal expression of multiple molecular markers were associated with an increased risk for cancer-specific mortality (multivariate analysis, $\mathrm{P}=0.029$ and 0.046, respectively). Clinocopathological characteristics, including gender, clinical stage, tumor size and number of tumors, were not associated with an increased risk for cancer-specific mortality by the Cox regression hazard model.

To investigate the potential association between abnormal expression of multiple biomarkers and the BCG response, we performed survival analysis for 44 patients who were

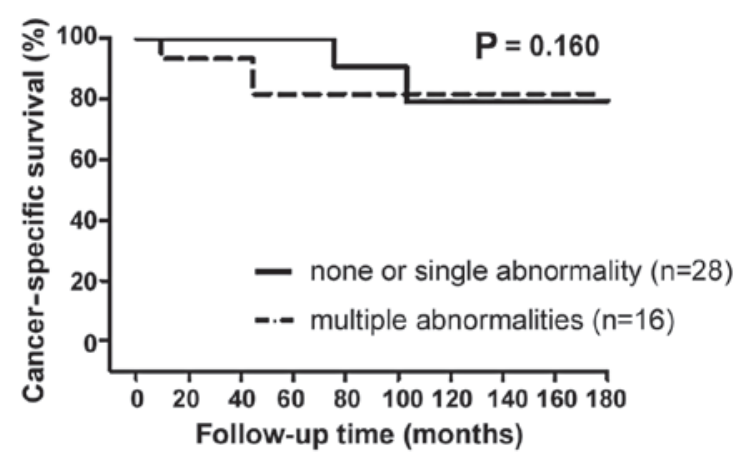

Figure 3. Probability of cancer-specific survival following initial transurethral resection according to the number of molecular markers abnormally expressed in patients treated with bacillus Calmette-Guérin (BCG) intravesical treatment.

administered BCG intravesical therapy. These patients were allocated into groups according to the number of markers abnormally expressed (0-1 vs. $\geq 2$ ). Analysis indicated no significant difference in CSS (log-rank test, $\mathrm{P}=0.160$ ) (Fig. 3) and the abnormal expression of multiple biomarkers did not predict the BCG response.

Of the 67 patients who initially retained their bladder, the abnormal expression of any single molecular marker and multiple biomarkers was not associated with an increased risk of bladder tumor recurrence when the log-rank test was performed (data not shown). 


\section{Discussion}

In the present study, the expression of E-cadherin, CAR, S100A4 and uroplakin III in patients diagnosed with high-grade NMIBC at initial TUR was assessed using immunohistochemistry and associated with clinical outcome. Our findings indicated that abnormal expression of $>1$ of these molecular markers is associated with an increased risk of bladder cancer-specific mortality. In the pathologically homogeneous group of patients with high-grade NMIBC, the number of abnormally expressed molecular markers varied from 0 to all the four markers. These findings suggest that high-grade NMIBC is characterized by a variety of pathophysiological pathways.

Distant metastases and progression to muscle invasion are the major causes of death in patients with high-grade NMIBC. Approximately $15-30 \%$ of patients exhibiting invasion into the lamina propria ultimately experience progression to muscle-invasive disease and $\sim 30 \%$ ultimately succumb to metastatic disease $(15,16)$. Aggressive treatments such as early cystectomy are recommended in some studies, since local invasion and distant metastasis would occur relatively early in patients diagnosed with high-grade NMIBC (17). By contrast, the lack of recurrence and progression of $\geq 30 \%$ of T1G3 tumors allows conservative management (1). In the present study, $45 \%$ of high-grade NMIBC patients were treated with conservative management and allowed bladder preservation without any evidence of tumor presence. Although early cystectomy might improve long-term survival (18), this aggressive approach might be an overtreatment in some cases. Many patients prefer to preserve the bladder and to be treated with a less invasive approach. Consequently, diagnostic approaches that distinguish aggressive diseases are needed. We evaluated the role of molecular markers as predictors of clinical outcome in high-grade NMIBC. We suggest that the abnormal expression of multiple molecular markers significantly increases the risk of cancer-specific mortality which is a useful criterion to distinguish patients with a poor prognosis. Physicians might provide better treatment options for individual patients diagnosed with high-grade NMIBC by utilizing these protein markers.

Other investigators have evaluated the clinical feasibility of biomarkers predicting high-grade NMIBC outcome. Park et al (19) examined whether altered protein expression for seven potential biomarkers, including p53, pRb, PTEN, Ki-67, p27, FGFR3 and CD9, was able to predict tumor progression in patients treated with BCG therapy for primary stage high-grade NMIBC. It was concluded that while increased p53 expression was associated with progression following BCG therapy, none of the remaining individual markers was associated with progression-free survival. Although p53 has been widely studied in this regard, the results were controversial $(20,21)$. Alvarez-Mugica et al (22) analyzed the methylation status of myopodin, a dual compartment protein that shows actin-bundling activity and is redistributed between the nucleus and cytoplasm in a differentiation-dependent, stress-induced manner. Myopodin methylation was shown to be associated with tumor aggressiveness and clinical outcome in patients with high-grade NMIBC. At present, only a few biomarkers have been suggested to be predictors of disease progression. These molecular markers are associated with angiogenesis, invasion and alterations in adhesion characteristics. However, their function is not well-understood. Further studies are needed to elucidate the individual mechanisms of these markers and their association to each other. This may lead to an understanding of how these markers contribute to tumor progression and metastasis, and facilitate clinical decision making.

BCG intravesical treatment is considered a standard treatment for decreasing tumor recurrence and delaying the progression of high-grade NMIBC. While this study suggested that BCG therapy improved CSS, the abnormal expression of multiple molecular markers lost its predictive significance in the subset of patients treated with BCG therapy. These results suggest that BCG therapy has beneficial effects on the survival of patients with abnormal expression of multiple biomarkers, who were found to have poor prognosis in the present study. However, the present study had certain limitations, including the fact that it was retrospective and that a relatively small number of patients were included. Additional prospective studies with larger numbers of patients are needed.

Second TUR and maintenance BCG therapy have been widely performed following diagnostic TUR in patients with high-grade NMIBC (23-26). These techniques were not used in the present study to improve outcomes since most of the included patients were diagnosed with high-grade NMIBC prior to the development of these techniques. These techniques are considered to decrease tumor recurrence and delay progression to muscle-invasive disease, while it is controversial whether they improve survival. The association of treatment modalities with molecular markers should be further investigated in future studies.

In conclusion, a combination of molecular markers plays a pivotal role in cancer progression, suggesting that high-grade NMIBC is characterized by a variety of pathophysiological pathways. Although no molecular markers are currently used in clinical practice, they have the potential to predict disease progression.

\section{References}

1. Shahin O, Thalmann GN, Rentsch C, Mazzucchelli L and Studer UE: A retrospective analysis of 153 patients treated with or without intravesical bacillus Calmette-Guerin for primary stage T1 grade 3 bladder cancer: recurrence, progression and survival. J Urol 169: 96-100, 2003.

2. Sylvester RJ, van der Meijden AP, Oosterlinck W, et al: Predicting recurrence and progression in individual patients with stage Ta T1 bladder cancer using EORTC risk tables: a combined analysis of 2596 patients from seven EORTC trials. Eur Urol 49: 466-465, 2006

3. Malavaud B: T1G3 bladder tumours: the case for radical cystectomy. Eur Urol 45: 406-410, 2004.

4. Turner W: T1G3 bladder tumours: the case for conservative treatment. Eur Urol 45: 401-405, 2004.

5. Agerbaek M, Alsner J, Marcussen N, Lundbeck F and Von der Maase H: Focal S100A4 protein expression is an independent predictor of development of metastatic disease in cystectomized bladder cancer patients. Eur Urol 50: 777-785, 2006.

6. Matsumoto K, Irie A, Satoh T, et al: Expression of S100A2 and S100A4 predicts for disease progression and patient survival in bladder cancer. Urology 70: 602-607, 2007.

7. Matsumoto K, Satoh T, Irie A, et al: Loss expression of uroplakin III is associated with clinicopathologic features of aggressive bladder cancer. Urology 72: 444-449, 2008.

8. Heimann R, Lan F, McBride R and Hellman S: Separating favorable from unfavorable prognostic markers in breast cancer: the role of E-cadherin. Cancer Res 60: 298-304, 2000. 
9. Andersen K, Nesland JM, Holm R, Florenes VA, Fodstad O and Maelandsmo GM: Expression of S100A4 combined with reduced E-cadherin expression predicts patient outcome in malignant melanoma. Mod Pathol 17: 990-997, 2004.

10. Okegawa T, Pong RC, Li Y, Bergelson JM, Sagalowsky AI and Hsieh JT: The mechanism of the growth-inhibitory effect of coxsackie and adenovirus receptor (CAR) on human bladder cancer: a functional analysis of car protein structure. Cancer Res 61: 6592-6600, 2001.

11. Sherbet GV and Lakshmi MS: S100A4 (MTS1) calcium binding protein in cancer growth, invasion and metastasis. Anticancer Res 18: 2415-2421, 1998.

12. Huang HY, Shariat SF, Sun TT, et al: Persistent uroplakin expression in advanced urothelial carcinomas: implications in urothelial tumor progression and clinical outcome. Hum Pathol 38: 1703-1713, 2007

13. Matsumoto K, Shariat SF, Ayala GE, Rauen KA and Lerner SP: Loss of coxsackie and adenovirus receptor expression is associated with features of aggressive bladder cancer. Urology 66 : 441-446, 2005

14. Matsumoto K, Kikuchi E, Horiguchi Y, et al: Late recurrence and progression in non-muscle-invasive bladder cancers after 5-year tumor-free periods. Urology 75: 1385-1390, 2010.

15. Zieger K, Wolf H, Olsen PR and Hojgaard K: Long-term survival of patients with bladder tumours: the significance of risk factors. Br J Urol 82: 667-672, 1998.

16. Millan-Rodriguez F, Chechile-Toniolo G, Salvador-Bayarri J, Palou J, Algaba F and Vicente-Rodriguez J: Primary superficial bladder cancer risk groups according to progression, mortality and recurrence. J Urol 164: 680-684, 2000.

17. van den Bosch S and Alfred Witjes J: Long-term cancer-specific survival in patients with high-risk, non-muscle-invasive bladder cancer and tumour progression: a systematic review. Eur Urol 60: 493-500, 2011.
18. Herr HW and Sogani PC: Does early cystectomy improve the survival of patients with high risk superficial bladder tumors? J Urol 166: 1296-1299, 2001.

19. Park J, Song C, Shin E, Hong JH, Kim CS and Ahn H: Do molecular biomarkers have prognostic value in primary T1G3 bladder cancer treated with bacillus Calmette-Guerin intravesical therapy? Urol Oncol: Jul 20, 2011 (Epub ahead of print).

20. Lacombe L, Dalbagni G, Zhang ZF, et al: Overexpression of p53 protein in a high-risk population of patients with superficial bladder cancer before and after bacillus Calmette-Guerin therapy: correlation to clinical outcome. J Clin Oncol 14: 2646-2652, 1996.

21. Schmitz-Drager BJ, Goebell PJ, Ebert T and Fradet Y: p53 immunohistochemistry as a prognostic marker in bladder cancer. Playground for urology scientists? Eur Urol 38: 691-699, 2000.

22. Alvarez-Mugica M, Cebrian V, Fernandez-Gomez JM, Fresno F, Escaf S and Sanchez-Carbayo M: Myopodin methylation is associated with clinical outcome in patients with T1G3 bladder cancer. J Urol 184: 1507-1513, 2010

23. Lamm DL, Blumenstein BA, Crissman JD, et al: Maintenance bacillus Calmette-Guerin immunotherapy for recurrent TA, T1 and carcinoma in situ transitional cell carcinoma of the bladder: a randomized Southwest Oncology Group Study. J Urol 163: 1124-1129, 2000.

24. Herr HW and Donat SM: A re-staging transurethral resection predicts early progression of superficial bladder cancer. BJU Int 97: 1194-1198, 2006.

25. Schwaibold HE, Sivalingam S, May F and Hartung R: The value of a second transurethral resection for T1 bladder cancer. BJU Int 97: 1199-1201, 2006.

26. Decobert M, LaRue H, Harel F, Meyer F, Fradet Y and Lacombe L: Maintenance bacillus Calmette-Guerin in high-risk nonmuscle-invasive bladder cancer: how much is enough? Cancer 113: 710-716, 2008. 